\title{
The Variability Of RSG : HV2576
}

\author{
Ming Yang and B. W. Jiang \\ Department of Astronomy, Beijing Normal University, Beijing, 100875, China \\ email: myang@mail.bnu.edu.cn and bjiang@bnu.edu.cn
}

\begin{abstract}
The Harvard Variable HV2576, as a red supergiant in the Large Magallanic Cloud, has a complex light variation, which is not explained well. The existing one-period non-linear pulsation model deviated clearly from its light curve. We tried to fit the light curve by a superposition of several harmonic pulsations. By using the PDM, PERIOD04 and SparSpec codes to analyze the light variation, two periods with 525 and 261 days are well established. Furthermore, the 261-day period is found to change according to the wavelet analysis. In addition, the noise obeying the $1 / \mathrm{f}$ law. Based on all these facts, we suggest that the light variation of HV2576 may be due to huge convection cells that interplay with oscillation or the third very long period.
\end{abstract}

Keywords. stars: oscillations, stars: supergiants, stars: variables: other

\section{Introduction}

Red supergiants (RSGs) are evolved, moderately massive $\left(10-30 M_{\odot}\right)$ He-burning stars. They have long been known for their brightness variation which is usually attributed to the radial pulsation. Moreover, an additional irregular variation occurs as well which may be caused by huge convection cell. The Harvard Variable HV2576 is a red supergiant in the Large Magallanic Cloud. It has a complex light variation. Wood (2006) tried to fit its light curve by a one-period, non-linear pulsation model, but the model deviates clearly from observed light curve. So we try to find out the reliable periods.

\section{Data Analysis}

The photometric data of $\mathrm{HV} 2576$ is taken from the MACHO database, which covers a duration of about 8 years. We used PERIOD04 (Lenz \& Breger 2005) to carry out standard iterative sine wave fitting (Fig. 1) together with the errorweighted analysis. From the power spectra, two distinct peaks are identified, corresponding to the periods 525 and 261 days with amplitude of $0.747 \mathrm{mag}$ and 0.751 mag respectively. There would be several more periods from the PERIOD04 analysis if only judged from the $\mathrm{S} / \mathrm{N}$ of the peaks. To clarify the true periods from spurious ones, the PDM (Phase Dispersion Minimization, Stellingwerf 1978) and SparSpect (Bourguignon, Carfantan, \& Bohm 2007) codes are used as well. Both

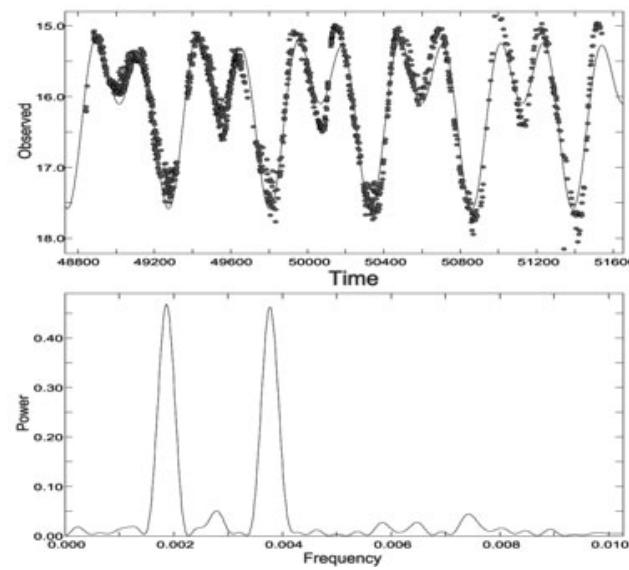

Figure 1: V-band light curve fitting with two periods and the power spectra derived from PERIOD04 
confirmed the 525-day period, which is consistent with the 530-day period obtained by Wood (2006). On the other hand, the 261-day period is present in these two methods as well, although it was not suggested in Wood (2006). From the consistency of these three methods, this 261-day period is taken as a real secondary period. Besides, the SparSpec code yields a third period of 174-day if error is weighted, but it is insignificant with unweighted error, neither in PERIOD04, thus abandoned.

With the two periods of 525-day and 261-day, the light curve is fitted as shown in Fig. 1, with the amplitude of $0.747 \mathrm{mag}$ and $0.751 \mathrm{mag}$ and the phase of 0.514 and 0.629 respectively. The fit is much improved than the one-period non-linear model, but still has some departure from the observed light curve. By eyes, the light curve shows a trend of change both in the amplitude and period. To check whether such trend is true, the WWZ (Weighted Wavelet Ztrasform) from AAVSO analysis is performed. The wavelet map shown in Fig. 2 again clearly re-confirm the two distinct periods derived above and indicate that the 261-day period has a trend to change. But the reason why the amplitude and period slowly grow

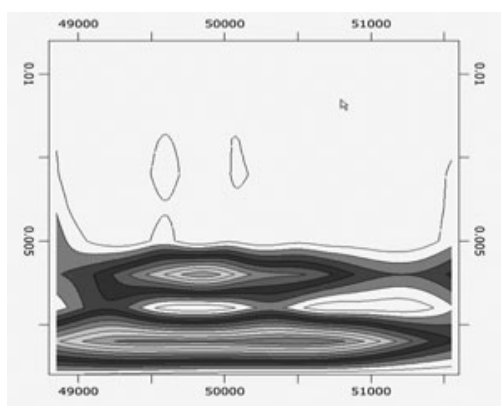

Figure 2: Contour wavelet map is not clear. One guess is that the convection or a third long period may lead to such situation.

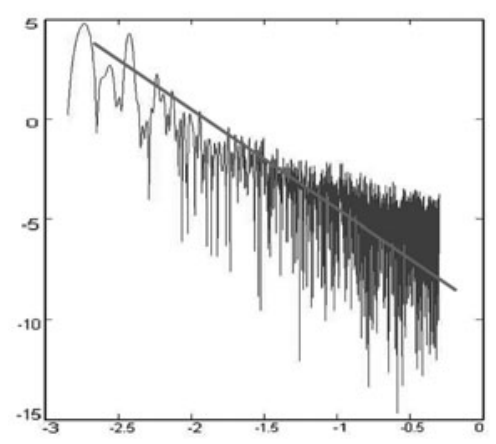

Figure 3: Power density spectrum in $\log$ $\log$ axis shows the $1 / \mathrm{f}$ noise
We also analyzed whether there is a $1 / \mathrm{f}$ noise component in the power spectra which imply the irregular variation owing to large convective cells, similarly to solar granulation background. In Fig. 3, we plot PDS in $\log -\log$ axis. The trend of $1 /$ f noise can be observed.

\section{Conclusion}

Analyzing the light curve of HV2576 with the powerful time-series analysis applications, two periods of 525 and 261 days are well established. In addition, the amplitude and period are found to vary. We suggest that the light variation of HV2576 may be due to huge convection cells that interplay with oscillation or the third long period.

\section{Acknowledgements}

This work is supported by the NSFC grant No.10778601.

\section{References}

Wood, P. R. 2006, Mem. S.A.It. 77, 76

Kiss, L. L., Szabo, Gy. M., \& Bedding, T. R. 2006, Mon. Not. R. Astron. Soc. 372, 1721

Templeton, M. 2004, JAAVSO 32, 41

Stellingwerf, R. F. 1978, ApJ 224, 953

Lenz, P. \& Breger, M. 2005, CoAst. 146, 53

Bourguignon, S., Carfantan, H., \& Bohm, T. 2007, AA 462, 379 